\title{
Spectroscopic investigations of corona discharge in high pressure Helium at $300 \mathrm{~K}$
}

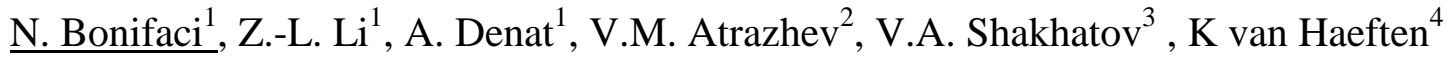 \\ ${ }^{1}$ Laboratoire G2Elab CNRS \& Joseph Fourier University. 25 rue des Martyrs, 38042 Grenoble, France \\ ${ }^{2}$ Joint Institute for High Temperatures, RAS, Moscow, 125412. \\ ${ }^{3}$ Topchiev of Petrochemical Synthesis Institute, RAS, Moscow, 119991. \\ ${ }^{4}$ University of Leicester Departement of Physics \& Asronomy United Kingdom
}

\begin{abstract}
Gaseous Helium at $300 \mathrm{~K}$ and pressure (0.1-3)MPa was excited using a corona discharge both for negative and positive high voltages. The light emitted from the ionization zone of the discharge was analyzed. The atomic lines observed was shifted and broadened due to interaction of radiators with surrounding atoms (the pressure broadening). Asymmetric shape of atomic line $706 \mathrm{~nm}$ was recorded. Blue wing of the line is more intensive than its red wing. The reason of the phenomenon is the repulsive interaction between excited atoms with atoms in the ground state. The line shape is described as a convolution of Lorentz profile of the line center and a quasi-statistical profile of a blue wing of the line. Such analysis allows us to predict a weak heating of the gas in the ionization zone for positive corona and considerable heating for negative corona.
\end{abstract}

\section{General}

Experiments have been carried out in gaseous $\mathrm{He}$ at the fixed temperature $300 \mathrm{~K}$ of the gas and different pressures in the cell from $0.1 \mathrm{MPa}$ up to $3 \mathrm{MPa}$. The corona discharge (ionization of gaseous $\mathrm{He}$ ) occurred in a vicinity of a tip electrode under high voltage. The discharge domain (ionization zone) had a volume less than an inter-electrode space (drift zone). The corona current has been measured for different temperatures and pressures in a space-charge-limited regime. This regime is characterized by an electric current as a quadratic function of the applied voltage $V$. Electrons with high mobility are the charge carriers in the drift region of the negative corona and it results in large negative corona current. Positive ions with low mobility are the charge carriers in the positive corona. Therefore the current of the positive corona is lower than that of the negative one.

The objective of the report is to predict the gas temperature in the ionization zone using the experimental data on spectral line emitted from the zone. It is shown that the gas is heated in the negative corona but not in the positive corona.

\section{Experimental technique}

The gaseous sample was produced from helium at the grade N 60 (Air Liquide) with an impurity concentration of about $0.1 \mathrm{ppm}$ of oxygen. The gas was further purified by a series of traps that were filled with a mixture of molecular sieves (3-10 $\AA$ ) and charcoal, activated under vacuum typically at $350^{\circ} \mathrm{C}$ for 3 days. The corona discharge cell included a point electrode and had a characteristic impedance of $50 \mathrm{Ohm}$ and it could withstand pressures up to $10 \mathrm{MPa}$. Before filling the cell was pumped to about $10^{-4} \mathrm{~Pa}$ using a turbo-molecular pump. Tungsten tips with a radius of $2.5 \mu \mathrm{m}$ were prepared by electrolytic etching. The electrode spacing was $8 \mathrm{~mm}$. All metallic electrodes were supported by Macor insulators. The high voltage from a stabilized dc power supply (Spellman RHSR/20PN60) was connected to the point electrode. In the cell the temperature of the gas could be adjusted to $300 \mathrm{~K}$ at a fixed pressure $P$ for each series of measurements.

Light emitted from the region close to the point electrode was analyzed by a spectrograph through a sapphire window. The spectrograph (Acton Research Corporation of $300 \mathrm{~mm}$ focal length) was equipped with 3 plane gratings: one with 150 $\mathrm{gr} . / \mathrm{mm}$ and two with $1200 \mathrm{gr} . / \mathrm{mm}$ that were blazed at $750 \mathrm{~nm}$ and $300 \mathrm{~nm}$, respectively. The 2DCCDTKB-UV/AR detector is located directly in the exit plane of the spectrograph. Its dimensions are $12.3 \times 12.3 \mathrm{~mm}$ with $512 \times 512$ pixels of $24 \times 24 \mu \mathrm{m}$ for each pixel. In order to reduce the dark current, the detector was cooled to a temperature of $153 \mathrm{~K}$ (dark current $<1 \mathrm{e} /$ pixel/hour at $153 \mathrm{~K}$ ). In our conditions, the instrumental broadening measured by recording profiles of argon lines from a low pressure discharge lamp is $\Delta \lambda_{\text {instr }}=0.1 \mathrm{~nm}$ for a 1200 grooves $/ \mathrm{mm}$ grating. 


\section{Experimental results}

The light emitted from the corona region was collected and spectra in the range $500-1080 \mathrm{~nm}$ were recorded. Figure 1 shows a representative spectrum observed. Most of atomic lines and molecular bands were identified. These lines correspond to radiative transitions between excited states of $\mathrm{He}^{*}$ atoms and $\mathrm{He}_{2}{ }^{*}$ excimer molecules.

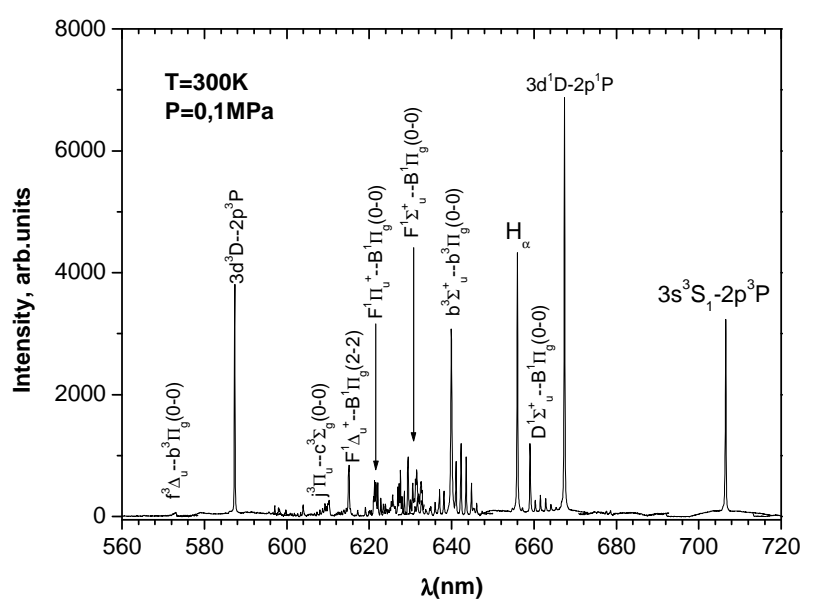

Fig.1. Overview spectrum recorded in the range 560-720 $\mathrm{nm}$ in Helium at $300 \mathrm{~K}$, and $0.1 \mathrm{MPa}$.

At low pressure the lines are sharp and their peak position match the atomic lines and molecular bands of helium from gas phase experiments. These lines are listed in Table 1.

Table 1. Transitions observed in Helium corona discharge (300K, $0.1 \mathrm{MPa})$

\begin{tabular}{|c|c|c|c|}
\hline \multicolumn{2}{|c|}{ Atomic lines } & \multicolumn{2}{|c|}{ Molecular bands } \\
\hline$\lambda(\mathrm{nm})$ & $\begin{array}{l}\text { Upper- } \\
\text { Lower }\end{array}$ & $\lambda(\mathrm{nm})$ & Upper-Lower \\
\hline 492,19 & $4 d^{1} D-2 p{ }^{1} P$ & 464,95 & $\mathrm{e}^{3} \Pi_{\mathrm{g}}-\mathrm{a}^{3} \Sigma_{\mathrm{u}}^{+}$ \\
\hline 587,56 & $3 d^{3} D-2 p{ }^{3} P$ & 573,49 & $\mathrm{f}^{3} \Delta_{\mathrm{u}}(\mathrm{v}=0)-\mathrm{b}^{3} \Pi_{\mathrm{g}}(\mathrm{v}=0)$ \\
\hline 706,52 & $3 s{ }^{3} S-2 p{ }^{3} P$ & 575 & $\begin{array}{l}\mathrm{f}^{3} \Delta_{\mathrm{u}}(\mathrm{v}=1)- \\
\mathrm{b}^{3} \Pi_{\mathrm{g}}(\mathrm{v}=1)\end{array}$ \\
\hline 728,13 & $3 s{ }^{1} S-2 p{ }^{1} P$ & 577 & $\begin{array}{l}f^{3} \Delta_{u}(v=2)- \\
b^{3} \Pi_{g}(v=2)\end{array}$ \\
\hline 1083,02 & $2 \mathrm{p}^{3} \mathrm{P}-2 \mathrm{~s}{ }^{3} \mathrm{~S}$ & 639,6 & $\mathrm{~d}^{3} \Sigma_{\mathrm{u}}^{+}-\mathrm{b}^{3} \Pi_{\mathrm{g}}$ \\
\hline & & 659,55 & $\mathrm{D}^{1} \Sigma_{\mathrm{u}}^{+}-\mathrm{B}^{1} \Pi_{\mathrm{g}}$ \\
\hline & & 913,61 & $\mathrm{C}^{1} \Sigma_{\mathrm{g}}^{+}-\mathrm{A}^{1} \Sigma_{\mathrm{u}}^{+}$ \\
\hline & & 918,3 & $\mathrm{c}^{3} \Sigma_{\mathrm{g}}^{+}-\mathrm{a}^{3} \Sigma_{\mathrm{u}}^{+}$ \\
\hline
\end{tabular}

A strong background continuum from 490 to 1100 $\mathrm{nm}$ appears in spectra at high pressures above $P=$ 4.0 MPa. Moreover, the width of the lines increases with pressure and their relative intensity decreases. The noise superimposes on the line shape at high pressures. Though, the qualitative characteristic of the line shape can be recorded up to $2.5 \mathrm{MPa}$. The small blue shifts of line maximum and more appreciable broadening of the lines are observed.

Atomic lines manifests asymmetric of their profile which increases with pressure. The skewness of the line shape is as larger intensity of its blue wing compare with a red wing. This effect is due to interaction of a radiator with surrounding atoms. The treatment of the phenomena is presented below and some properties of the corona ionization zone is predicted based on the treatment.

In this report we concentrate our attention on the analysis of shape of the atomic line $706 \mathrm{~nm}$. Figures 2 and 3 show the atomic $3 s^{3} \mathrm{~S}->2 p^{3} \mathrm{P}$ line at $706 \mathrm{~nm}$ being broadened and shifted with increasing pressure towards smaller wavelengths (blue shift).

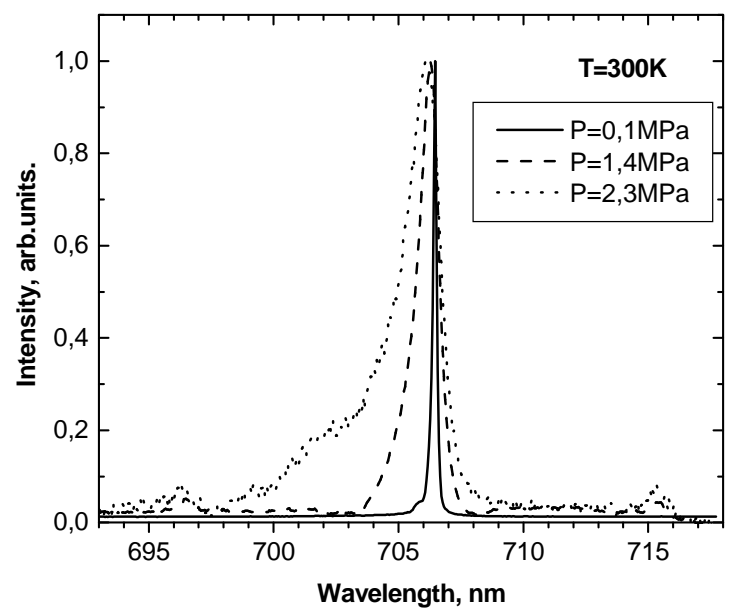

Fig.1. Experimental spectra obtained for $706 \mathrm{~nm}$ line in negative corona under different pressures.

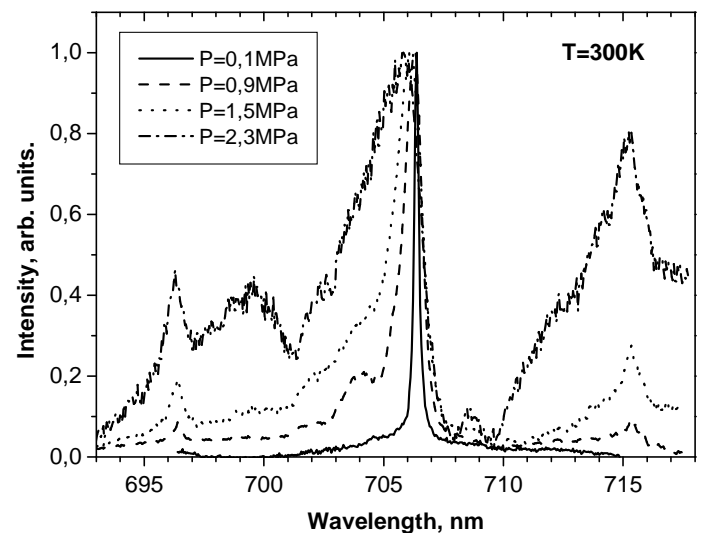

Fig.2. Experimental spectra obtained for $706 \mathrm{~nm}$ line in positive corona under different pressures.

The shapes of the line observed in negative and positive corona are different, but both spectra exhibit the blue shift and more intensive blue wing. The figures present very interesting phenomenon of "satellites" observed at both $695 \mathrm{~nm}$ and $716 \mathrm{~nm}$. 
Their intensity increases with pressure and the effect is more appreciable in the positive corona. The phenomena of the "satellites" will be considered in our future work. Here we analyze the shape of the central line and give an explanation in the framework of the classic theory of pressure broadening of spectral lines.

\section{Theoretical treatment}

The theory of "impact" broadening of spectral lines predicts symmetric Lorentzian profile of the line with shift and width being proportional to a gas density $N$ [1]. The analysis of the line distortion due to model Lennard-Jones potential of the interaction between radiator and perturbators [2] showed that the shift of the line depends on the nature of the interaction. If the van der Waals attraction prevails over the short range repulsion, the shift is toward long-length side. If the repulsion is prevailed, the "blue" shift is predicted. The blue shift has been observed in our experiments and this allows us to assume the repulsive interaction $\left(U(r)=C_{12} r^{-12}\right)$ between a radiator and perturbators. Within the "impact" approximation for the repulsive interaction, the expressions for the line broadening $\Delta \lambda$ (Full Width at Half Maximum) and the line shift $S$ are given by:

$$
\begin{aligned}
& \Delta \lambda=6.44\left(\frac{\lambda^{2}}{2 \pi c}\right) w^{9 / 11}\left|C_{12}\right|^{2 / 11} N \\
& S=0.922\left(\frac{\lambda^{2}}{2 \pi c}\right) w^{9 / 11}\left|C_{12}\right|^{2 / 11} N
\end{aligned}
$$

Here $\lambda=706 \mathrm{~nm}$ is the wavelength of the line, $w=$ $10^{4} * T^{0.5}$ is the relative velocity in $[\mathrm{cm} / \mathrm{s}]$ of the gas atoms with $T$ being the gas temperature in $[\mathrm{K}], C_{12}$ is the repulsive Lennard-Jones parameter in $\left[\mathrm{cm}^{12} \mathrm{~s}^{-1}\right]$ and $N$ is the gas number density in $\left[\mathrm{cm}^{-3}\right]$. The collision approximation predicts a ratio of the shift and the width of 0.143 which is indeed close to what we find in our experiments with $\mathrm{He}$ gas at $300 \mathrm{~K}$. The theoretical calculations with more realistic description of the interaction than L-J potential [3], gave for the $706 \mathrm{~nm}$ line shift and broadening at $300 \mathrm{~K}$

$$
\begin{aligned}
& \Delta \lambda=2.9 \cdot 10^{-21} \cdot N \\
& S=0.384 \cdot 10^{-21} \cdot N
\end{aligned}
$$

Using the theoretical value for the broadening (2) and its treatment with the repulsive potential (1) one can estimate the repulsion constant value $C_{12}=$ $1.6 * 10^{-72} \mathrm{~cm}^{12} / \mathrm{s}$.

It has been shown in [4] that the asymmetric line can be obtained in the "statistical" approximation in the frame of the pressure broadening theory. The approximation assumes zero velocity of atoms and it becomes significant for large density of a gas. The profile of a line $I(\omega)$ as a function of detuning $\Delta \omega$ from the line center $\Delta \omega=0$ is described by the formula [5]

$$
I(\Delta \omega)=\frac{1}{2 \pi} \int_{-\infty}^{\infty} \exp [i \Delta \omega \rho-4 \pi N V(\rho)] d \rho
$$

Here a red wing corresponds to $\bullet \omega<0$ and the blue wing is $\bullet \omega>0$. The interaction between the radiator and perturbators $\Delta U(R)$ as a function of their spacing $R$ is described by the term $V(\rho)$

$V(\rho)=\int_{0}^{\infty}\left[1-\exp \left(-i \rho \frac{\Delta U(R)}{\hbar}\right)\right] R^{2} d R$

The property $V(-\rho)=V^{*}(\rho)$ allows us to rewrite Eq.(3) in the form [3]

$I(\Delta \omega)=\frac{1}{2 \pi} \int_{0}^{\infty}\left(\begin{array}{l}\exp \left[-i \Delta \omega \rho-4 \pi N V^{*}(\rho)\right]+ \\ +\exp [i \Delta \omega \rho-4 \pi N V(\rho)]\end{array}\right) d \rho$

Below we shall use the simple form of the repulsive potential $\Delta U(R)=C_{12} / R^{12}$ with one parameter $C_{12}$. It allows us to describe the line profile using dimensionless variables

$I_{\text {wing }}(x)=\int_{0}^{\infty} \exp (-2.41 y) \cdot \cos \left(x \cdot y^{4}-y\right) y^{3} d y$

Here $x=\frac{\Delta \omega}{15.02 \cdot N^{4} C_{12}}$. The integral in Eq.(5) differs from zero for $x>0$ (blue wing of the line), Fig.3.

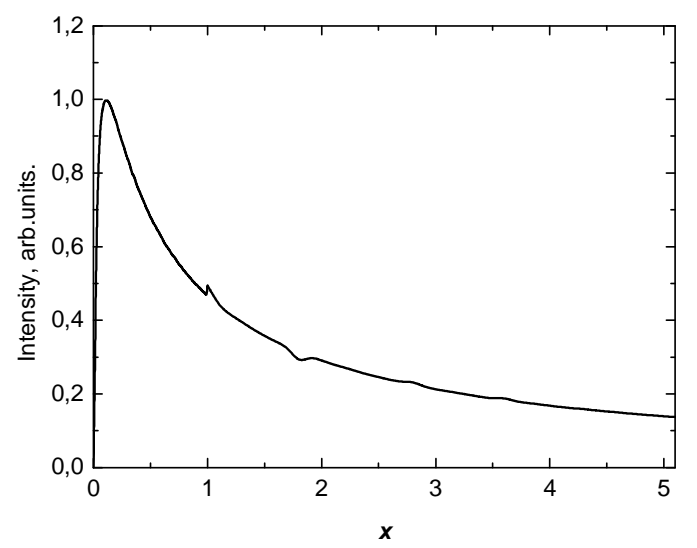

Fig.3. Profile of a line $I_{\text {wing }}(x)$ in the frame of the statistical approximation for $C_{12} / R^{12}$ repulsive potential as a function of the dimensionless frequency.

The statistical profile has the blue shift and describes the long frequency wing of the line. The center of the line is described by the Lorentzian profile with small shift $S$ and the width $\Delta \lambda$ according to Eq.(2) 


$$
I_{L}(\lambda)=\frac{\left(\frac{\Delta \lambda}{2}\right)^{2}}{\left(\left(\lambda-\lambda_{0}+S\right)^{2}+\left(\frac{\Delta \lambda}{2}\right)^{2}\right)}
$$

In order to combine the central profile Eq.(7) and the wing-profile Eq.(6), the convolution operation was used in a manner as it been done in [6]

$$
I(\lambda)=\int_{0}^{\lambda_{0}} I_{\text {wing }}(x) I_{L}(\lambda-x) d x
$$

Here the integration variable $x$ is the difference between the wing wavelength and the wavelength of the line center $\lambda_{0}, x=\left(\lambda_{0}-\lambda\right)>0$. The analytical approximation

$$
I_{\text {wing }}(x)=\exp \left(-0.1 \frac{\Delta \lambda_{w}}{x}\right)\left(\frac{x}{\Delta \lambda_{w}}\right)^{-0.9} \quad x>0 .
$$

was used for the wing statistical profile Eq.(6), where the parameter of the statistical approximation

$$
\Delta \lambda_{w}=\frac{15.02 \lambda_{0}{ }^{2} N^{4} C_{12}}{2 \pi c}=2.48 \cdot 10^{-6}\left[\mathrm{~nm} / \mathrm{bar}^{4}\right] \cdot p^{4}
$$

has strong density dependence as $N^{4}$. Using the analytical form for $I_{\text {wing }}(x)$ one can obtain the expression for the total shape of the line, Eq.(8) as

$$
I(\lambda)=\int_{0}^{\infty} \frac{z^{0.9} \exp (-0.1 \cdot z)}{\left.\left(\Delta \lambda_{w}+\lambda \cdot z\right)^{2}+\left(\frac{\Delta \lambda}{2}\right)^{2} \cdot z^{2}\right)} d z
$$

Here $\lambda$ is a wavelength from the shifted center of the line, $\lambda_{0}-S$. The total profile depends on the impact broadening of line center (FWHM is $\Delta \lambda$, Eq.(2)) and on the statistical parameter $\Delta \lambda_{\mathrm{w}}$ Eq.(10). These values have different density dependence.

Figures 4 and 5 show the result of calculation of the 706nm line shape for pressures $1.5 \mathrm{MPa}$ and 2.3 MPa using Eq.(11) observed in positive and negative coronas.

For simulation of the line for the positive corona the gas density was calculated using the pressure values and the temperature $300 \mathrm{~K}$ of the ionization zone of the discharge. The accordance between experiment and simulation shows that there is no heating of the gas in corona zone in the positive corona.

The line observed in the negative corona has less broadening than predicted by its simulation using the gas density calculated using the pressure values and the temperature $300 \mathrm{~K}$ of the ionization zone of the discharge.

The most adequate agreement between the experimental line profiles and their simulation occurs if the temperature $500 \mathrm{~K}$ is assumed for the ionization zone of the negative corona. Indeed, the negative corona currant in the space-charge-limited conditions is larger than that in the positive corona, because higher electron mobility in the drift zone of the negative corona. The larger current results in possibility of considerable heating of the gas near a tip electrode.

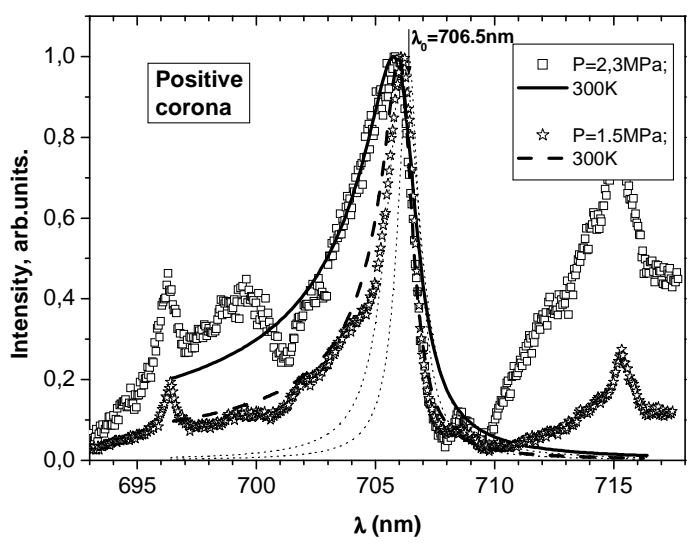

Fig.4. Simulation of the $706 \mathrm{~nm}$ line emitted by positive corona at $300 \mathrm{~K}$ and $1.5 \mathrm{MPa}$ and $2.3 \mathrm{MPa}$ - solid and dashed lines; dotted lines - Lorentzian profiles.

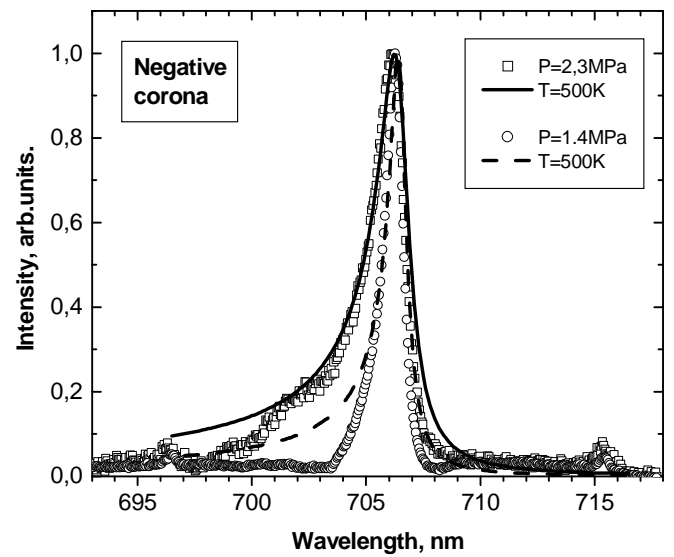

Fig.5. Simulation of the $706 \mathrm{~nm}$ line emitted by negative corona at $500 \mathrm{~K}$ and $1.5 \mathrm{MPa}$ and $2.3 \mathrm{MPa}$ - solid and dashed lines.

Authors from Russia (V.M.A. and V.A.S.) thank Russian Foundation of Basic Researches for support of their work; grant 08-08-00694

\section{References}

[1] G. Traving Interpretation of line broadening and line shift in Plasma Diagnostic (Chap. 2), edited by W. Lochte-Holtgreven, North-Holland Publishing Company, Amsterdam (1968). 
[2] W. R. Hindmarsh, A. D. Petford,. G. Smith, Proc. Roy. Soc., A297, 296 (1967)

[3] P. J. Leo, et.al., J. Phys. B, 25, 1161 (1992)

[4] H. Margenau, Phys. Rev., 48, 755 (1935)

[5] N. Allard, J. Kielkopf, Rev. Mod. Phys., 54, 1103 (1982)

[6] H.-P. Stormberg, J. Appl. Phys., 51, 1963 (1980) 